\title{
Prognostic value of AIBI and EIF5A2 in intravesical recurrence after surgery for upper tract urothelial carcinoma
}

This article was published in the following Dove Press journal:

Cancer Management and Research

\author{
Yong Huang ${ }^{1,2, *}$ \\ Jinhuan Wei ${ }^{1, *}$ \\ Yong Fang ${ }^{1, *}$ \\ Zhenhua Chen',* \\ Junjie Cen ${ }^{1}, *$ \\ Zihao Feng' \\ Jun Lu' \\ Yanping Liang' \\ Junhang Luo' \\ Wei Chen' \\ 'Department of Urology, The First \\ Affiliated Hospital, Sun Yat-sen \\ University, Guangzhou 510080, China; \\ ${ }^{2}$ Department of Emergency, The \\ First Affiliated Hospital, Sun Yat-sen \\ University, Guangzhou 510080, China \\ *These authors contributed equally to \\ this work
}

Correspondence: Wei Chen

Department of Urology, The First

Affiliated Hospital, Sun Yat-sen

University, No 58, Zhongshan 2nd Road,

Guangzhou 510080, China

Tel +862087755766

Fax +862087333300

Email chenwei3sysu@163.com
Objectives: The aim of this study was to investigate the prognostic effect of amplified in AIB1 and EIF5A2 expression on postoperative intravesical recurrence for upper urinary tract urothelial carcinoma (UTUC) and improve postoperative risk stratification and prediction of intravesical chemotherapy benefit.

Materials and methods: We evaluated immunohistochemical expression of AIB1 and EIF5A2 in 109 UTUC patients to determine the predictive significance in intravesical recurrence. A prognostic model was developed based on univariate and multivariate analyses.

Results: Intravesical recurrence occurred in 18 out of the 109 (16.5\%) patients during the follow-up period. Significant associations of high expression of AIB1 and EIF5A2 with shortened bladder recurrence interval (median: 24 months vs 46 months, $P=0.021 ; 28$ months vs 39 months, $P=0.002)$ were demonstrated. In different subsets of UTUC patients, high expression of AIB1 was a prognostic indicator in high grade $(P=0.006)$ and pT2-4 $(P=0.007)$, and high expression of EIF5A2 for high grade $(P=0.014)$, pT2 $4(P=0.002)$ and pN0 $(P=0.009)$. Moreover, in multivariate analysis, AIB1 and EIF5A2 expression ( $P=0.034$ and 0.022 , respectively) together with $\mathrm{pN}$ stage $(P=0.009)$ provided significant independent predictors for intravesical recurrence after surgery for UTUC. Surgical approach with radical nephroureterectomy (RNU) was an informative factor toward good oncologic outcomes for intravesical recurrence $(P=0.056)$. Based on a prognostic model with these factors, patients with UTUC were classified into the low-risk group and the high-risk group. In a subset analysis, the patients in the high-risk group were found to have a favorable response to intravesical chemotherapy $(P=0.047)$. A nomogram based on the multivariate analysis was developed to predict intravesical recurrence accurately and guide postoperative intravesical instillations. The concordance index (c-index) of this model was 0.806 .

Conclusion: High expression of AIB1 and EIF5A2 were independent predictors for intravesical recurrence after RNU and might be able to predict which patients benefit from postoperative intravesical chemotherapy.

Keywords: AIB1, EIF5A2, intravesical recurrence, prognosis, nomogram, upper tract urothelial carcinoma

\section{Introduction}

Urothelial carcinoma (UC) refers to tumors derived from epithelial cells lining the urinary tract, and it can be divided into upper tract urothelial carcinoma (UTUC) and bladder urothelial carcinoma (BUC). ${ }^{1}$ Although UTUC accounts for $8 \%$ of the renal tumors and $2 \%-5 \%$ of the $\mathrm{UCs},{ }^{2}$ up to $23 \%-47.2 \%$ of the patients may suffer bladder recurrence after surgery for UTUC..$^{3-5}$ At present, the main variables defining prognosis are histopathological stage and grade, but patients with the same pTNM 
stage of UTUC often demonstrate considerable variability in bladder recurrence. ${ }^{6}$ Thus, many research studies on UTUC try to establish the role of molecular elements in predicting the postoperative outcome in the case of this neoplasm. ${ }^{7-9}$ To date, an ideal biomarker for the prediction of disease recurrence is still lacking.

The amplified in breast cancer 1 (AIB1) gene, also named as SRC3, p/CIP, RAC3, ACTR and TRAM1, is located on chromosome $20 \mathrm{q} 12 .{ }^{10} \mathrm{AIB} 1$, as a human oncogene, has been found to be involved in a number of biological processes, such as cell proliferation, cell migration and cell differentiation. ${ }^{10,11}$ AIB1 was reported to promote proliferation in hormone-dependent cancers, ${ }^{10,12,13}$ and AIB1 upregulation was associated with tumor aggressiveness and/or poor patient prognosis in several human hormone-independent cancers. ${ }^{14-17}$ Previously, we demonstrated that AIB1 is an independent molecular marker for poor prognosis of BUC patients. $^{18}$

The eukaryotic initiation factor 5A2 (EIF5A2) gene was first discovered in the primary ovarian cancer cell line in 2001. It was located on chromosome 3 q26 and also classified as an oncogene. ${ }^{19}$ Overexpression of EIF5A2 was reported to predict poor prognosis in gastric adenocarcinomas, ${ }^{20}$ colorectal cancer, ${ }^{21}$ hepatocellular carcinomas, ${ }^{22}$ ovarian cancer $^{23}$ and non-small-cell lung cancer. ${ }^{24}$ Our previous study showed that EIF5A2 was an independent molecular marker for shortened survival time of BUC patients treated with radical cystectomy. ${ }^{25}$

Although UTUC usually shares histologic appearance with BUC, recent studies have reported that the biology of these 2 entities differs, and these differences may affect different genetic presentations. ${ }^{26,27} \mathrm{Up}$ to the present, the molecular state of AIB1 and EIF5A2 gene in UTUC and the prognostic significance of this state have not been examined. In this study, immunohistochemistry (IHC) was used to examine protein expression of AIB1 and EIF5A2 in a group of human UTUCs. The primary purpose was to determine whether or not AIB1 and EIF5A2 activity has prognostic value for bladder tumor recurrence in UTUC patients. In addition, a tool for guidance of a risk-stratified approach to postoperative surveillance could be developed if possible.

\section{Materials and methods}

\section{Study design and participants}

We reviewed the records of 133 patients with primary UTUC from September 2000 to March 2016 in the First Affiliated Hospital of Sun Yat-sen University. The patients did not receive any chemotherapy or radiation therapy before surgery. The patients with distant metastasis at diagnosis and incomplete clinical data were excluded from this study. In 24 tissues from UTUC patients the detection of AIB1 or EIF5A2 was not completed, and so the remaining 109 patients were included in this study. Radical nephroureterectomy (RNU) with the removal of the bladder cuff was the most common procedure $(\mathrm{n}=95)$. Segmental ureterectomy (SU) was performed in 12 patients, and kidney-sparing surgery (KSS) was performed in 2 patients. Regional lymphadenectomy was generally performed in patients with suspicious lymph nodes on preoperative imaging or intraoperative examination. Extended lymphadenectomy was not routinely performed. Eighty-seven patients received intravesical instillation after surgery, and the intravesical agents were epirubicin (30-50 mg each time, 55 cases), pirarubicin (30-50 mg each time, 19 cases) or mitomycin C (MMC) (20-40 mg each time, 13 cases). At least 6 doses of intravesical chemotherapy weekly were recommended after surgery. Cystoscopy was suggested every 3 months for 2 years and every 6 months for the next 2 years and annually thereafter. Other follow-ups consisted of physical examination, urine cytology, chest X-ray and computed tomography. The study methodologies conformed to the standards set by the Declaration of Helsinki. Approval of the study was obtained from the institutional review board of the First Affiliated Hospital, Sun Yat-sen University, and written informed consent was waived as there were no conflicts of interest or damage to patients, and patient data confidentiality was guaranteed according to the requirement of the institutional review board. The tissue samples were also de-identified due to the subsequent copy, cryopreservation and storage time in this retrospective study.

\section{IHC}

Formalin-fixed paraffin-embedded tissue samples were used for IHC studies. Serial $4 \mu \mathrm{m}$ sections were prepared for immunohistochemical staining. The IHC method was similar to that used in our previous study. ${ }^{18}$ Briefly, after paraffin sections were deparaffinized in xylene and rehydrated in a series of graded alcohols, $0.3 \%$ hydrogen peroxide was used to block endogenous peroxidase activity for 20 minutes, and then tissue sections were microwave-treated and boiled in a $10 \mathrm{mmol} / \mathrm{L}$ citrate buffer ( $\mathrm{pH}$ 6.0) for 8 minutes for antigen retrieval. Non-specific binding was blocked with $10 \%$ normal rabbit serum for 20 minutes. The slides were incubated with the anti-AIB1 antibody (BD Transduction Laboratories; Lexington, KY, USA; diluted 1:100 in PBS) or anti-EIF5A2 antibody (Abnova, Jhongli, Taiwan; diluted 1:100 in PBS) in a moist chamber. Subsequently, the slides were incubated with biotinylated rabbit anti-mouse immunoglobulin at a con- 
centration of $1: 100$ for 30 minutes at $37^{\circ} \mathrm{C}$ and then reacted with a streptavidin-peroxidase conjugate for 30 minutes at $37^{\circ} \mathrm{C}$ and $3^{\prime}, 3^{\prime}$-diaminobenzidine as a chromogen substrate. The nucleus was counterstained using Meyer's hematoxylin. A negative control was obtained by replacing the primary antibody with normal murine IgG. Known immunostainingpositive ovarian cancer slides were used as positive controls.

Positive expression of AIB1 in UTUC cells was primarily a nuclear pattern, and positive expression of EIF5A2 was a cytoplasmic pattern. Semiquantitative assessment of positivity was performed using the following formula: staining intensity $\times$ positive cell rate. Cancer cells with positive staining were counted in at least 10 representative fields, and at least 150 cancer cells were examined for each field. Staining intensity was stratified from 0 to 3 ( 0 , no staining; 1 , slight staining; 2 , medium staining; and 3, strong staining). The scale of positive cell rate was identified as follows: 0 , number of stained cells $<10 \% ; 1,10 \leq$ number of stained cells $<25 \% ; 2,25 \% \leq$ number of stained cells $<50 \% ; 3,50 \% \leq$ number of stained cells $<80 \%$; and 4 , number of stained cells $\geq 80 \%{ }^{7,28} \mathrm{We}$ selected the medians as the cutoff value (score range $0-12$ ), respectively. An AIB1 expression score greater than 4 was considered high AIB1 expression, and an EIF5A2 expression score greater than 6 was defined high EIF5A2 expression.

\section{Statistical analyses}

Analysis was carried out with SPSS version 20.0 (IBM Corporation, Armonk, NY, USA) and GraphPad Prism 5 (GraphPad Software, Inc., La Jolla, CA, USA). The association between AIB1 and EIF5A2 and clinicopathological features was assessed by the chi-squared test. Clinical outcome event was intravesical recurrence-free survival (IVRFS) obtained by Kaplan-Meier actuarial analysis. IVRFS was defined as the time from surgery to intravesical recurrence. The association between each prognostic factor and IVRFS was assessed by univariate analysis. A stepwise selection procedure was applied to obtain a multivariate model with maximum precision for the significant variables. To predict recurrence-free survival probability, multivariable Cox regression coefficients were then used to generate a nomogram and calibration curves with R for Windows, version 3.3.1 (R Foundation for Statistical Computing, Vienna, Austria). For validation of the nomogram, we assessed the discrimination ability using Harrell's concordance index (c-index). All $P$-values were 2 -sided, and $P$-values $<0.05$ were considered to be significant.

\section{Results}

A total of 109 patients with primary UTUC were included in this cohort. The median age was 66 years (range
21-86). The main clinical data are detailed in Table 1. The male/female ratio was $1.48: 1$. The follow-up time was $44.8 \pm 35.2$ months, and the median was 34 months (range 1-180). Intravesical recurrence of bladder cancer occurred in 18 out of $109(16.5 \%)$ patients, and the follow-up time was $38.2 \pm 50.3$ months, and the median was 16 months (range 1-180). Relapse was observed in $9(50.0 \%)$ patients with renal pelvis location and in $6(33.3 \%)$ patients with ureteral location.

All the specimens were fixed in 10\% formalin and embedded in paraffin. To elucidate the biological significance of

Table I Clinicopathological features of patients with UTUC

\begin{tabular}{|c|c|}
\hline Clinicopathological features & $N=109$ \\
\hline Median age, years (range) & $66(21-86)$ \\
\hline \multicolumn{2}{|l|}{ Sex, n (\%) } \\
\hline Male & $65(59.6)$ \\
\hline Female & $44(40.4)$ \\
\hline \multicolumn{2}{|l|}{ Tumor side, $\mathrm{n}(\%)$} \\
\hline Left & $54(49.5)$ \\
\hline Right & $55(50.5)$ \\
\hline \multicolumn{2}{|l|}{ Tumor location, n (\%) } \\
\hline Calix or pelvis & $60(55.0)$ \\
\hline Ureter & $38(34.9)$ \\
\hline$>$ I site & $11(10.1)$ \\
\hline \multicolumn{2}{|l|}{ Hydronephrosis, n (\%) } \\
\hline No & $34(3 \mid .2)$ \\
\hline Yes & $75(68.8)$ \\
\hline \multicolumn{2}{|l|}{ Type of surgery, $n(\%)$} \\
\hline Open & $55(50.5)$ \\
\hline Laparoscopic & $54(49.5)$ \\
\hline \multicolumn{2}{|l|}{ RNU, n (\%) } \\
\hline No & 14 (| 2.8$)$ \\
\hline Yes & $95(87.2)$ \\
\hline \multicolumn{2}{|l|}{ Pathological T stage, n (\%) } \\
\hline $\mathrm{Ta}-\mathrm{TI}$ & $37(33.9)$ \\
\hline $\mathrm{T} 2-\mathrm{T} 4$ & $72(66.1)$ \\
\hline \multicolumn{2}{|l|}{ Pathological N stage, n (\%) } \\
\hline $\mathrm{pNx}$ or $\mathrm{pN} 0$ & $93(85.3)$ \\
\hline $\mathrm{pNI}, 2$ & $16(14.7)$ \\
\hline \multicolumn{2}{|l|}{ Tumor grade, $\mathrm{n}(\%)$} \\
\hline Low & $38(34.9)$ \\
\hline High & $71(65.1)$ \\
\hline \multicolumn{2}{|l|}{ Intravesical chemotherapy, n (\%) } \\
\hline No & $22(20.2)$ \\
\hline Yes & $87(79.8)$ \\
\hline \multicolumn{2}{|l|}{ Adjuvant chemotherapy, n (\%) } \\
\hline No & $83(76.1)$ \\
\hline Yes & $26(23.9)$ \\
\hline \multicolumn{2}{|l|}{ AIBI, n (\%) } \\
\hline Low expression & $58(53.2)$ \\
\hline High expression & $51(46.8)$ \\
\hline \multicolumn{2}{|l|}{ EIF5A2, n (\%) } \\
\hline Low expression & $61(56.0)$ \\
\hline High expression & $48(44.0)$ \\
\hline
\end{tabular}

Abbreviations: RNU, radical nephroureterectomy; UTUC, upper tract urothelia carcinoma. 
AIB1 and EIF5A2 in UTUC, IHC was performed on all slides using anti-AIB1 and anti-EIF5A2. We set the cutoff points at 4 and 6 based on the median score of AIB1 and EIF5A2 scores, respectively, and patients were divided into low AIB1 ( $\mathrm{n}=58)$ and high AIB1 expression groups $(\mathrm{n}=51)$ and low EIF5A2 ( $\mathrm{n}=61)$ and high EIF5A2 expression groups $(n=48)$ in order to analyze their oncologic outcomes (Figure 1). The association of AIB1 and EIF5A2 expression with clinicopathological features in UTUCs is summarized in Table 2. Among the features, there was a significant association between AIB1 expression and intravesical therapy $(P=0.006)$, and EIF5A2 expression was significantly higher in UTUC with positive lymph node $(P=0.007)$.

Median IVRFSs in the low and high AIB1 expression groups were observed to be 46 months (range 6-140) and 24 months (range 1-180), respectively. Statistically significant differences were observed between the low and high AIB1 expression groups in the 5-year IVRFS rate $(90.5 \%$ vs $76.5 \%, P=0.021)$. The survival intervals in the low and high EIF5A2 expression groups were 39 months (range 2-131) and 28 months (range 1-180), respectively. The 5-year IVRFS rates of the low and high EIF5A2 expression groups were $94.2 \%$ and $72.6 \%(P=0.002)$, as shown in Figure 2 .
Additionally, survival analysis was performed with regard to AIB1 and EIF5A2 expression in subsets of patients with different tumor histopathological grades, $\mathrm{pT}$ stages and $\mathrm{pN}$ stages. The results demonstrated that high expression of AIB1 and EIF5A2 were poor prognostic factors in UTUC patients having high tumor grade ( $P$-value: 0.006 and 0.014 , respectively) and pT2-4 ( $P$-value: 0.007 and 0.002 , respectively). In addition, EIF5A2 expression showed a statistical significance with regard to the prognosis of pN0 patients $(P=0.009)$. However, either AIB1 or EIF5A2 expression could not differentiate the outcome of $\mathrm{pN} 1-2$ patients ( $P$-value: 0.056 and 0.787; Figures 3 and 4).

To investigate the effect of intravesical chemotherapy, survival analysis was also performed in subsets of patients with different AIB1 and EIF5A2 expression. The results showed that intravesical chemotherapy was a good prognostic factor for IVRFS of UTUC patients with high AIB1 and EIF5A2 expression ( $P=0.002$; Figure 5$)$. In addition, patients with pT2-4 (16/20, 80\%), pN+ (7/20,35\%) or high grade (13/20, $65 \%$ ) were included in this subset.

Univariate and multivariate analyses of conventional clinicopathological factors and molecular markers were carried out to analyze the risk factors affecting intravesical recurrence
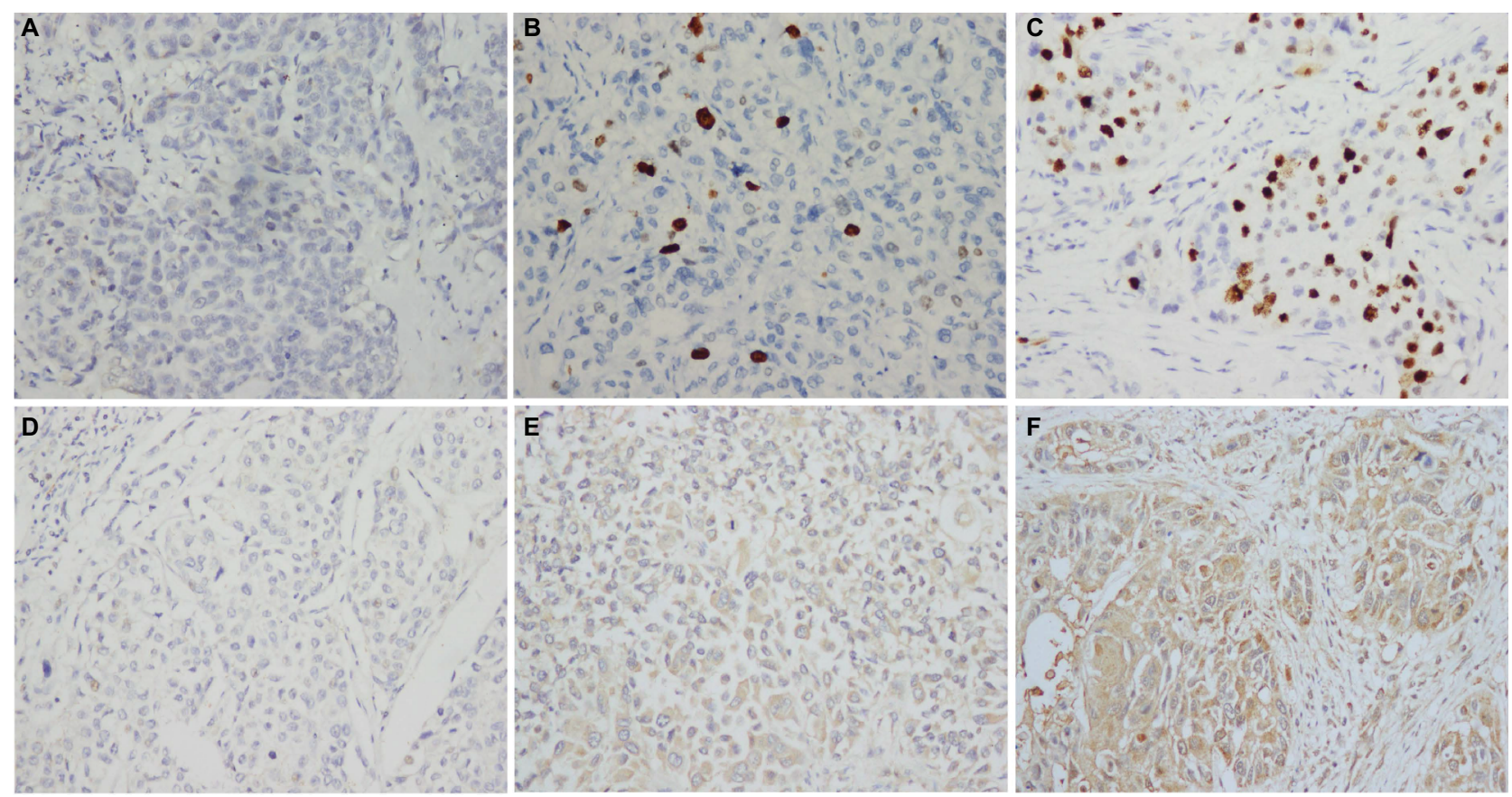

Figure I Immunohistochemical expression of AIBI and EIF5A2 in upper tract urothelial carcinoma tissue $(\times 200)$.

Notes: (A) AIBI score 0 points (negative staining), (B) low expression of AIBI (score 3 points), (C) high expression of AIBI (score 9 points), (D) EIF5A2 score 0 points (negative staining), (E) low expression of EIF5A2 (score 4 points) and (F) high expression of ElF5A2 (score 12 points). 
Table 2 Association of AIBI and EIF5A2 expression with clinicopathological parameters in 109 patients with UTUC

\begin{tabular}{|c|c|c|c|c|c|c|}
\hline \multirow[t]{2}{*}{ Variables } & \multicolumn{2}{|l|}{ AIB I } & \multirow[t]{2}{*}{$P$-value } & \multicolumn{2}{|l|}{ EIF5A2 } & \multirow[t]{2}{*}{$P$-value } \\
\hline & $\begin{array}{l}\text { Low } \\
\text { expression }\end{array}$ & $\begin{array}{l}\text { High } \\
\text { expression }\end{array}$ & & $\begin{array}{l}\text { Low } \\
\text { expression }\end{array}$ & $\begin{array}{l}\text { High } \\
\text { expression }\end{array}$ & \\
\hline \multicolumn{7}{|l|}{ Age, years } \\
\hline$\leq 66$ & 30 & 26 & 0.938 & 27 & 29 & 0.094 \\
\hline$>66$ & 28 & 25 & & 34 & 19 & \\
\hline \multicolumn{7}{|l|}{ Sex } \\
\hline Male & 38 & 27 & 0.182 & 39 & 26 & 0.302 \\
\hline Female & 20 & 24 & & 22 & 22 & \\
\hline \multicolumn{7}{|l|}{ Tumor side } \\
\hline Left & 29 & 25 & 0.919 & 26 & 28 & 0.103 \\
\hline Right & 29 & 26 & & 35 & 20 & \\
\hline \multicolumn{7}{|l|}{ Tumor location } \\
\hline Calix or pelvis & 32 & 28 & 0.885 & 32 & 28 & 0.669 \\
\hline Ureter & 20 & 19 & & 24 & 15 & \\
\hline >I site & 6 & 4 & & 5 & 5 & \\
\hline \multicolumn{7}{|l|}{ Hydronephrosis } \\
\hline No & 21 & 13 & 0.228 & 15 & 19 & 0.093 \\
\hline Yes & 37 & 38 & & 46 & 29 & \\
\hline \multicolumn{7}{|l|}{ Type of surgery } \\
\hline Open & 25 & 30 & 0.101 & 27 & 28 & 0.145 \\
\hline Laparoscopic & 33 & 21 & & 34 & 20 & \\
\hline \multicolumn{7}{|l|}{ RNU } \\
\hline No & 6 & 8 & 0.406 & 8 & 6 & 0.924 \\
\hline Yes & 52 & 43 & & 53 & 42 & \\
\hline \multicolumn{7}{|l|}{ Pathological T stage } \\
\hline $\mathrm{Ta}-\mathrm{TI}$ & 21 & 16 & 0.595 & 22 & 15 & 0.598 \\
\hline $\mathrm{T} 2-\mathrm{T} 4$ & 37 & 35 & & 39 & 33 & \\
\hline \multicolumn{7}{|l|}{ Pathological N stage } \\
\hline $\mathrm{pNx}$ or $\mathrm{pN} 0$ & 53 & 40 & 0.057 & 57 & 36 & $0.007^{*}$ \\
\hline $\mathrm{pNI}, 2$ & 5 & 11 & & 4 & 12 & \\
\hline \multicolumn{7}{|l|}{ Tumor grade } \\
\hline Low & 20 & 18 & 0.929 & 23 & 15 & 0.483 \\
\hline High & 38 & 33 & & 38 & 33 & \\
\hline \multicolumn{7}{|c|}{ Intravesical chemotherapy } \\
\hline No & 6 & 16 & $0.006 *$ & 11 & 11 & 0.528 \\
\hline Yes & 52 & 35 & & 50 & 37 & \\
\hline \multicolumn{7}{|c|}{ Adjuvant chemotherapy } \\
\hline No & 49 & 34 & $0.029 *$ & 45 & 38 & 0.512 \\
\hline Yes & 9 & 17 & & 16 & 10 & \\
\hline \multicolumn{7}{|l|}{ AlBI } \\
\hline Low expression & - & - & - & 30 & 28 & 0.342 \\
\hline High expression & - & - & & 31 & 20 & \\
\hline
\end{tabular}

Note: *Statistically significant.

Abbreviations: RNU, radical nephroureterectomy; UTUC, upper tract urothelial carcinoma.

(Table 3). In univariate analysis, lymph node metastasis and high-level AIB1 and EIF5A2 expression were significantly associated with intravesical recurrence $(P<0.05)$. Multivariate Cox proportional hazards regression analysis revealed that lymph node metastasis $(P=0.009)$, high-level AIB1 expression $(P=0.034)$ and high-level EIF5A2 expression $(P=0.022)$ were significant risk factors for IVRFS. Surgical approach with RNU ( $P=0.056)$ was an informative protective factor, but not an independent factor.
We constructed a prognostic model combining 4 independent prognostic factors (EIF5A2, AIB1, lymph node status and RNU) by Cox proportional hazards regression. The model for IVRFS $=$ EIF5A2 score $\times 1.529+$ AIB1 score $\times 1.179+\mathrm{pN}$ status $\times 1.538-\mathrm{RNU} \times 1.151$. According to the median of the model to construct the risk stratification, we defined 2 risk groups, the low-risk group and the highrisk group. Results showed that patients in the low-risk group had a much better 5-year IVRFS rate than that in the 
A

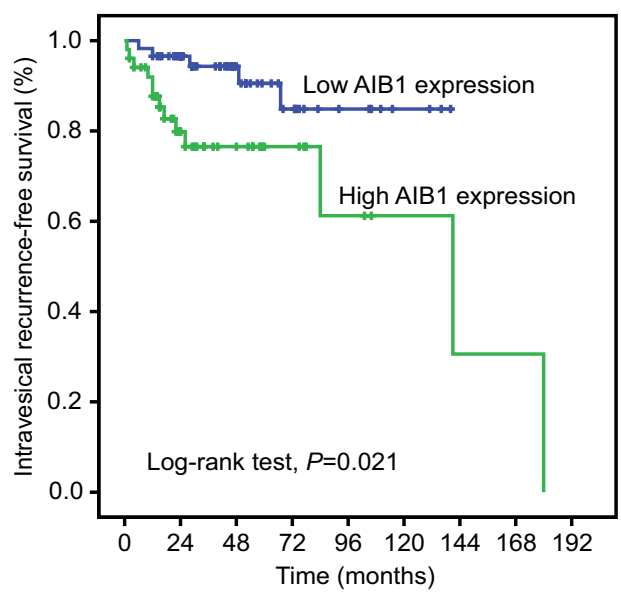

B

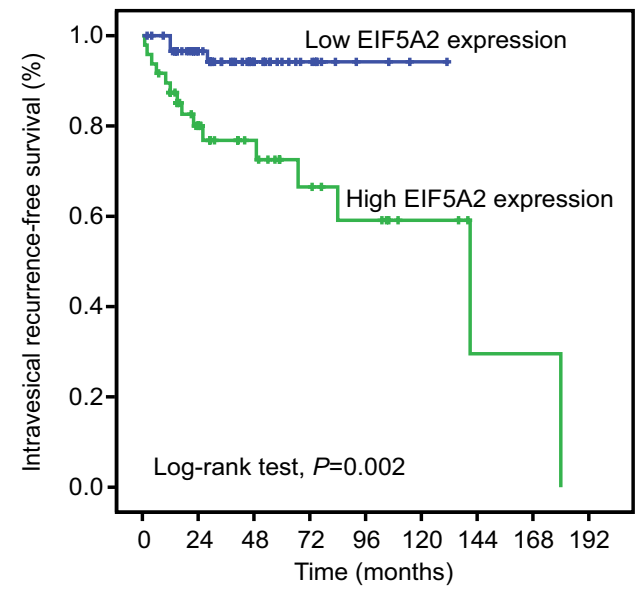

Figure 2 Kaplan-Meier plots show intravesical recurrence-free survival curves according to $\mathrm{AIBI}$ expression status (A), low $\mathrm{AIBI}$ expression (blue line), $\mathrm{n}=58$; high $\mathrm{AIBI}$ expression (green line), $n=51$. EIF5A2 expression status (B), low EIF5A2 expression (blue line), $n=61$; high EIF5A2 expression (green line), $n=48$.

high-risk group (94.1\% vs 60.6\%; Figure S1). We noted that intravesical chemotherapy could enhance survival in all 109 patients $(P=0.047)$. Results from a subset analysis using the prognostic model showed that patients in the high-risk group had a favorable response to intravesical chemotherapy ( $P=0.047$; Figure 6). This result indicated that this prognostic model could successfully identify patients with UTUC who were suitable candidates for intravesical chemotherapy.

Receiver operating characteristic (ROC) curve analysis was performed to determine the predictive value of the prognostic model including AIB1 and EIF5A2. The result showed that the prognostic model had significantly higher prognostic accuracy than that of a single clinicopathological risk factor (Figure 7). The ROC curves yielded the following area under curve (AUC): AIB1, 0.652, 95\% CI (0.517-0.788); EIF5A2, $0.735,95 \%$ CI (0.617-0.854); pN status, $0.612,95 \% \mathrm{CI}$ (0.457-0.767); surgical approach (RNU or not), $0.411,95 \%$ CI (0.256-0.565) and the prognostic model, $0.824,95 \% \mathrm{CI}$ (0.714-0.934).

A competing-risk nomogram was developed for the prediction of the probability of postoperative recurrence in bladder at 2 years and 5 years. This model was based on AIB1, EIF5A2, pathologic $\mathrm{N}$ status and the surgical approach (RNU or not). By calculating the total number of risk points on the nomogram, one is able to calculate the 2-year and 5-year probabilities of intravesical recurrence after surgery. The calibration plots were separately demonstrated for a 2-year IVRFS probability and 5-year
IVRFS probability (Figure 8), and the c-index of this model was 0.806 .

\section{Discussion}

The key findings in this study are that high expressions of AIB1 and EIF5A2 are strongly linked with intravesical recurrence after operation in patients with UTUC, especially in the subsets of high grade and high pathological stage. Furthermore, both of them are independent predictors of bladder recurrence as evidenced by Kaplan-Meier curves and multivariate $\mathrm{COX}$ proportional hazards regression analysis. Although the mechanisms of carcinogenesis are thought to be similar to bladder cancer, recent studies suggest otherwise. UTUCs seem to present worse clinical outcomes than bladder cancers at the same stage. ${ }^{29}$ Despite UTUC accounts for $5 \%-10 \%$ of UC, the incidence of UTUC has increased during the last 30 years, and so it is meaningful to focus on small samples of UTUCs to explore the difference with bladder tumors. ${ }^{30,31}$ Our previous studies have already shown that overexpression of AIB1 and EIF5A2 contributes to shortened survival time of patients with bladder cancers. In another study, it has been demonstrated that AIB1 promotes bladder cancer cell proliferation through the AKT pathway and E2F1.32 From this study, we hypothesize that AIB1 and EIF5A2 play an important role in UTUC. Until now, no study has been found to investigate the predictive value of the combination of both markers.

Some studies had observed that in certain human solid tumors, such as ovarian and colorectal cancers, high levels 
A

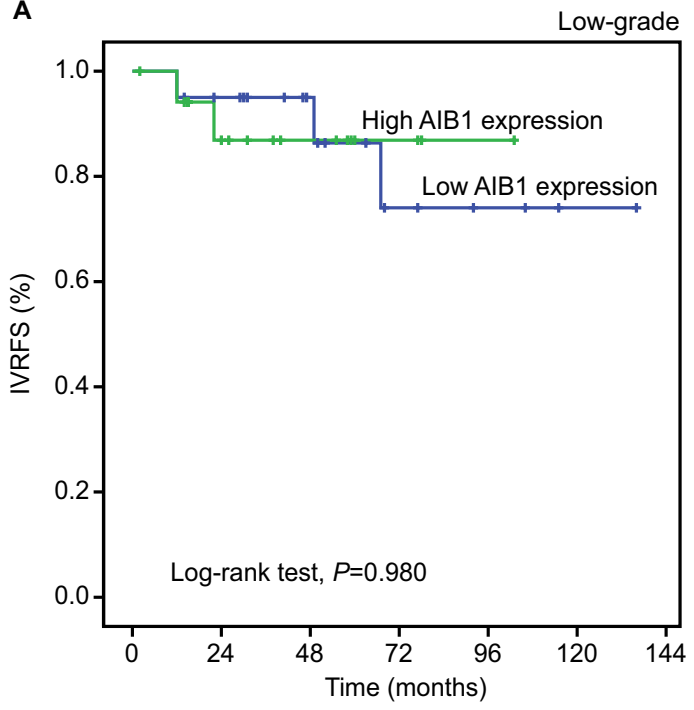

C

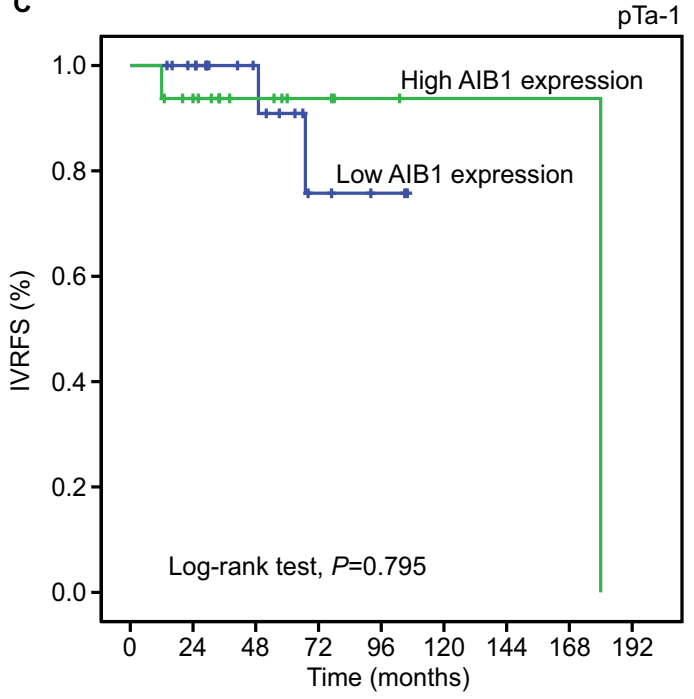

E

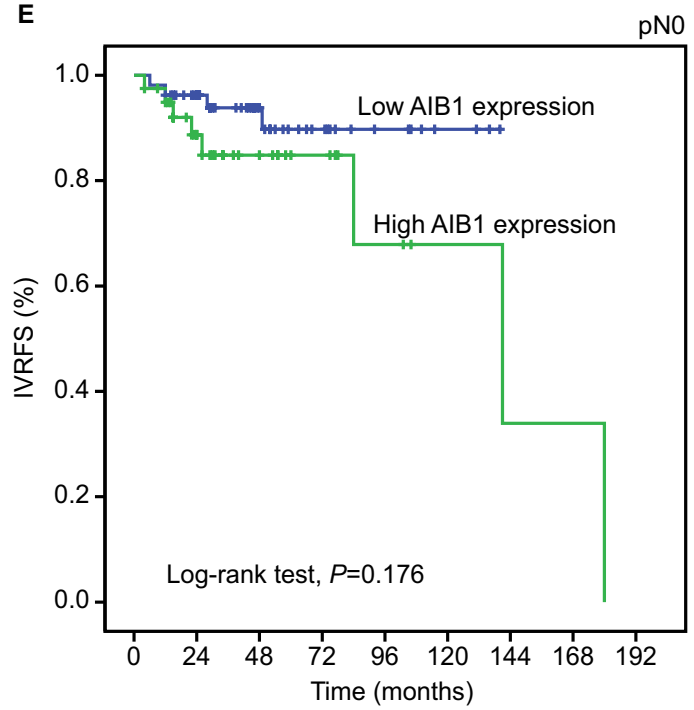

B

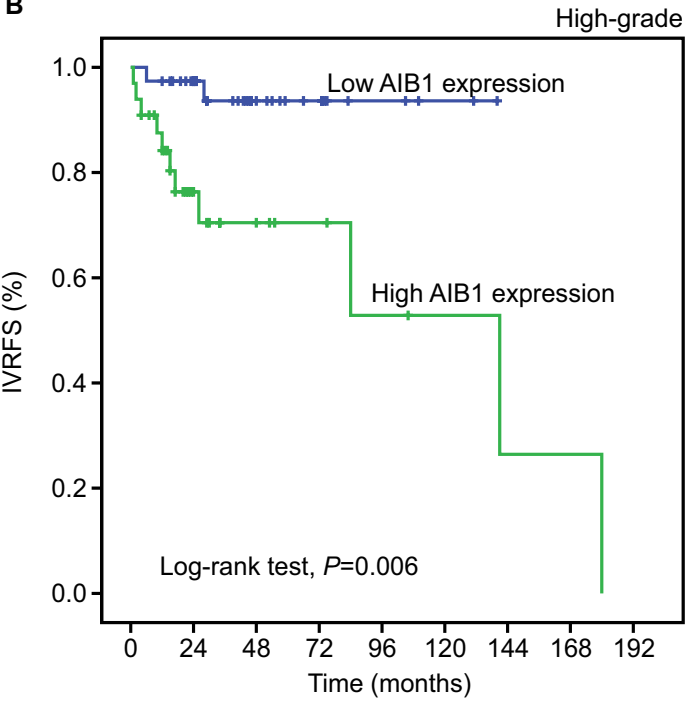

D

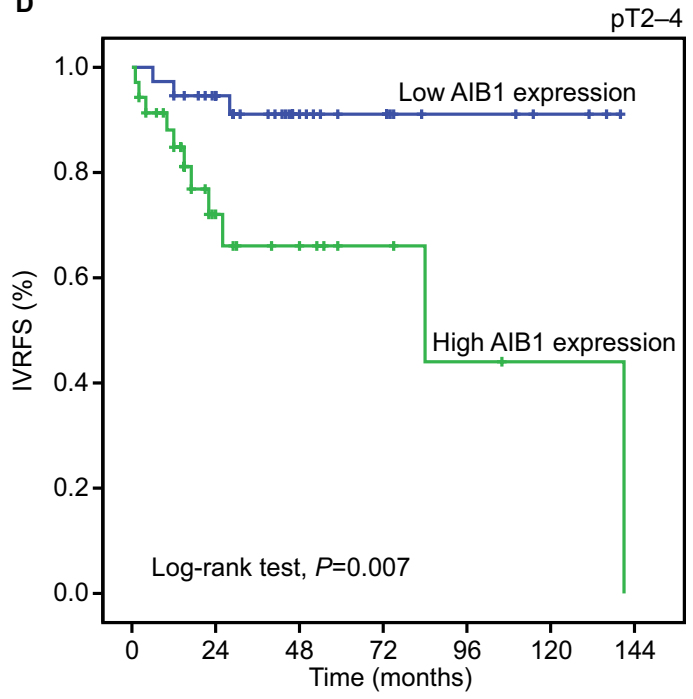

$\mathbf{F}$

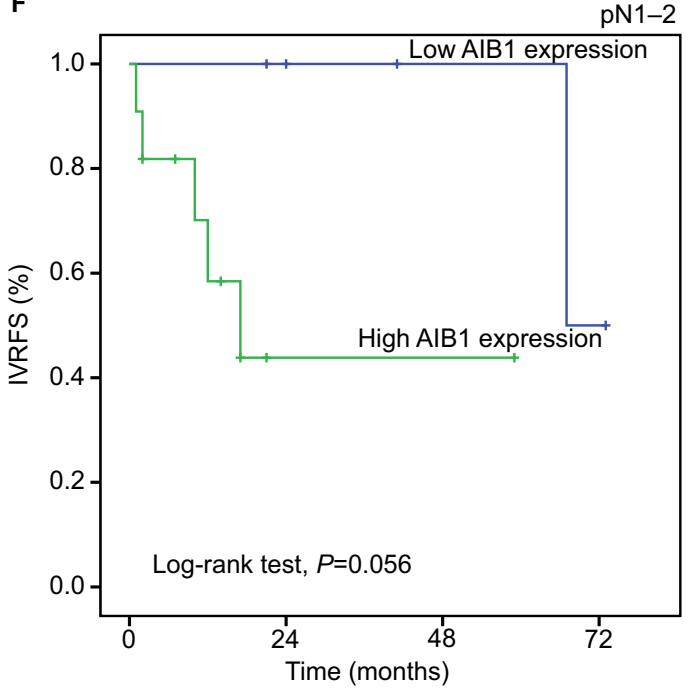

Figure 3 Kaplan-Meier survival analysis of AIBI expression in subsets of different grades, $\mathrm{pT}$ and $\mathrm{pN}$ patients with UTUC (log-rank test).

Notes: (A) Low-grade, probability of IVRFS of low-grade patients with UTUC: low expression (blue line), n=20; high expression (green line), $n=18$. (B) High-grade, probability of IVRFS of high-grade patients with UTUC: low expression (blue line), $\mathrm{n}=38$; high expression (green line), $\mathrm{n}=33$. (C) pTa-I, probability of IVRFS of pTa-I patients with UTUC: low expression (blue line), $n=21$; high expression (green line), $n=16$. (D) PT2-4, probability of IVRFS of pT2-4 patients with UTUC: low expression (blue line), $n=37$; high expression (green line), $n=35$. (E) pNO, probability of IVRFS of pN0 patients with UTUC: low expression (blue line), $n=53$; high expression (green line), $n=40$. (F) pNI-2, probability of IVRFS of pNI-2 patients with UTUC: low expression (blue line), $n=5$; high expression (green line), $n=1$.

Abbreviations: IVRFS, intravesical recurrence-free survival; UTUC, upper tract urothelial carcinoma. 
A

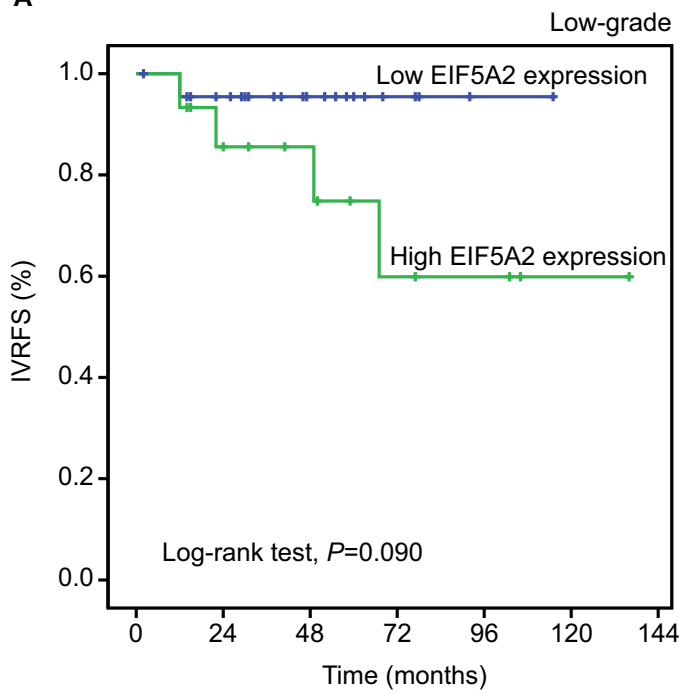

C

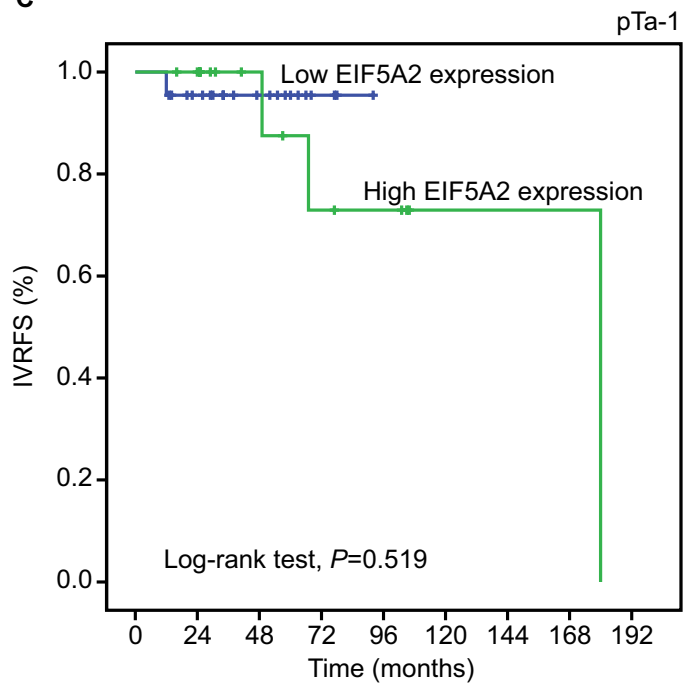

E

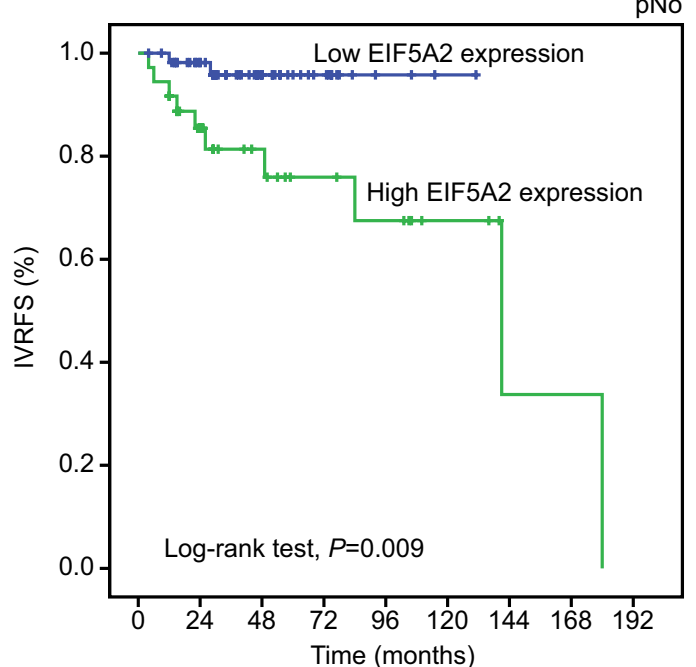

B

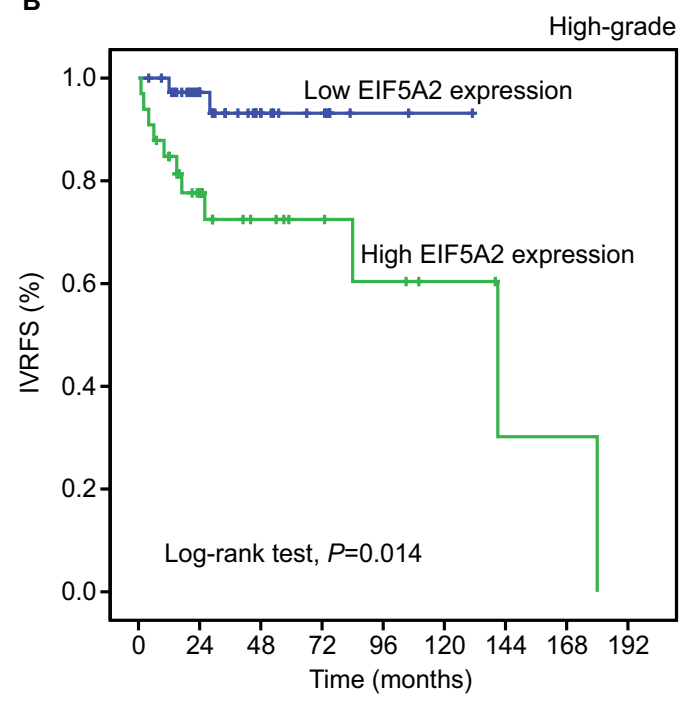

D

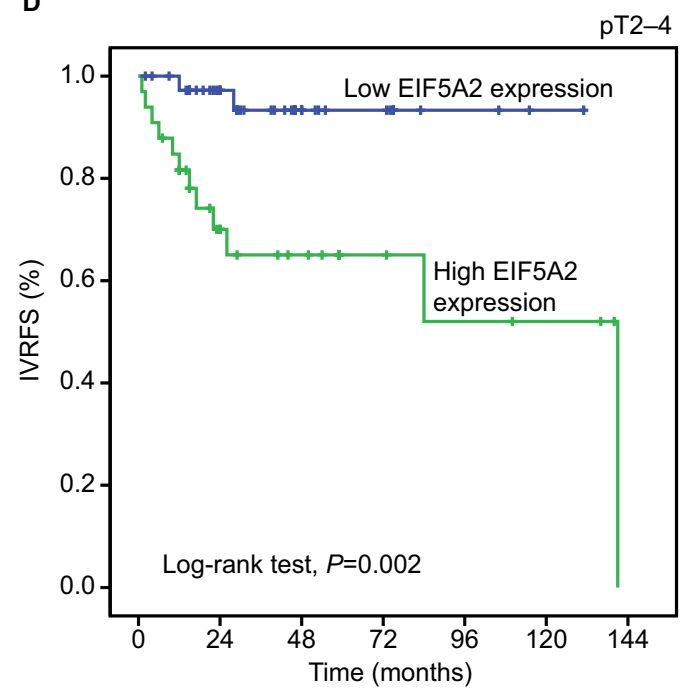

$\mathbf{F}$

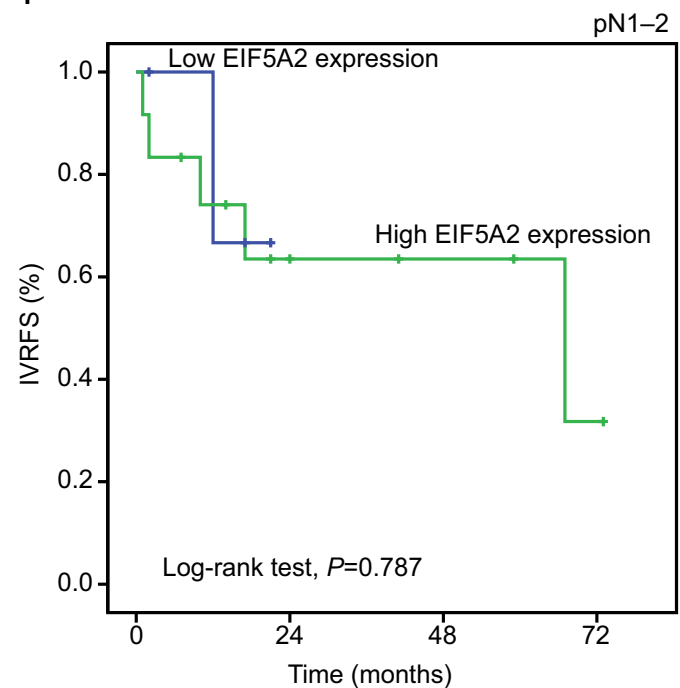

Figure 4 Kaplan-Meier survival analysis of EIF5A2 expression in subsets of different grades, pT and pN patients with UTUC (log-rank test).

Notes: (A) Low-grade, probability of IVRFS of low-grade patients with UTUC: low expression (blue line), $n=23$; high expression (green line), $n=15$. (B) High-grade, probability of IVRFS of high-grade patients with UTUC: low expression (blue line), n=38; high expression (green line), $n=33$. (C) pTa-I, probability of IVRFS of pTa-I patients with UTUC: low expression (blue line), $n=22$; high expression (green line), $n=15$. (D) pT2-4, probability of IVRFS of pT2-4 patients with UTUC: low expression (blue line), $n=39$; high expression (green line), $n=33$. (E) pN0, probability of IVRFS of pN0 patients with UTUC: low expression (blue line), $n=57$; high expression (green line), $n=36$. (F) $\mathrm{pNI}-2$, probability of IVRFS of $\mathrm{pNI}-2$ patients with UTUC: low expression (blue line), $n=4$; high expression (green line), $n=12$.

Abbreviations: IVRFS, intravesical recurrence-free survival; UTUC, upper tract urothelial carcinoma. 
A

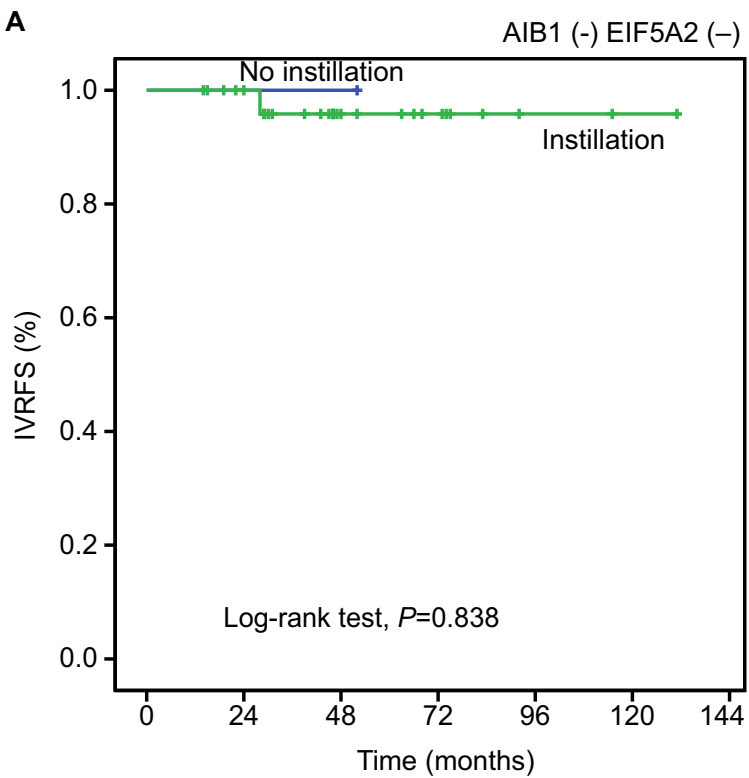

C

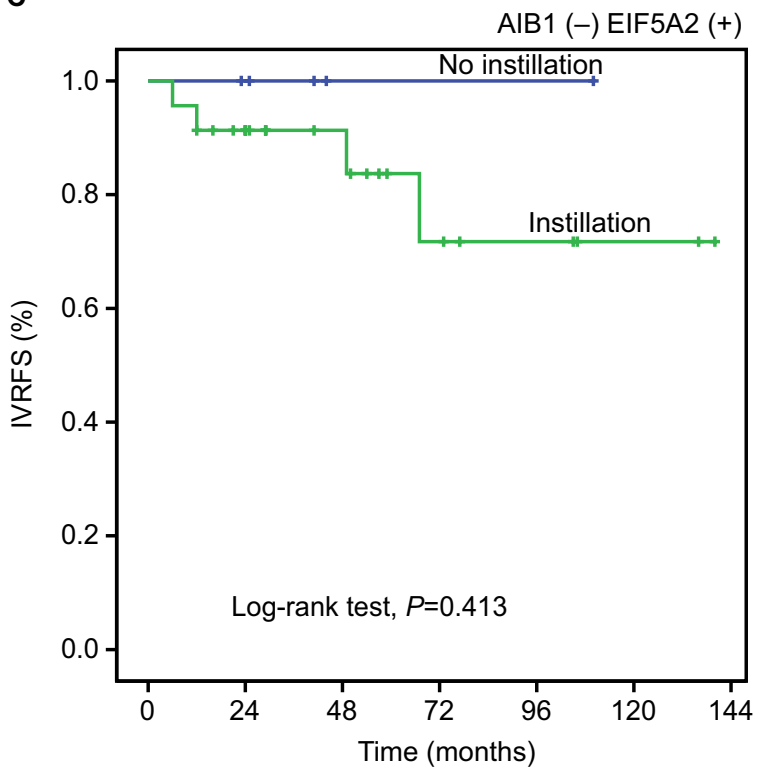

B

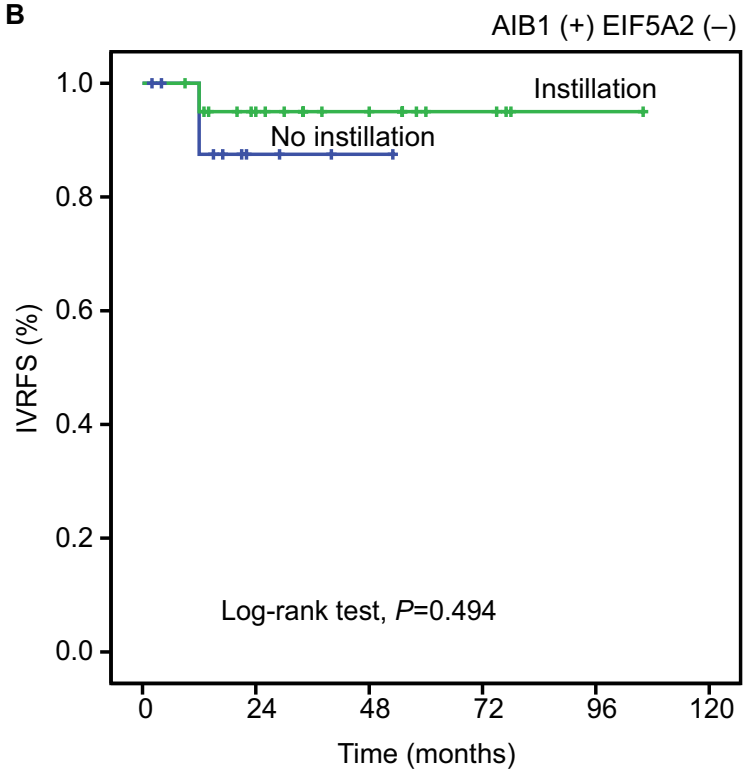

D

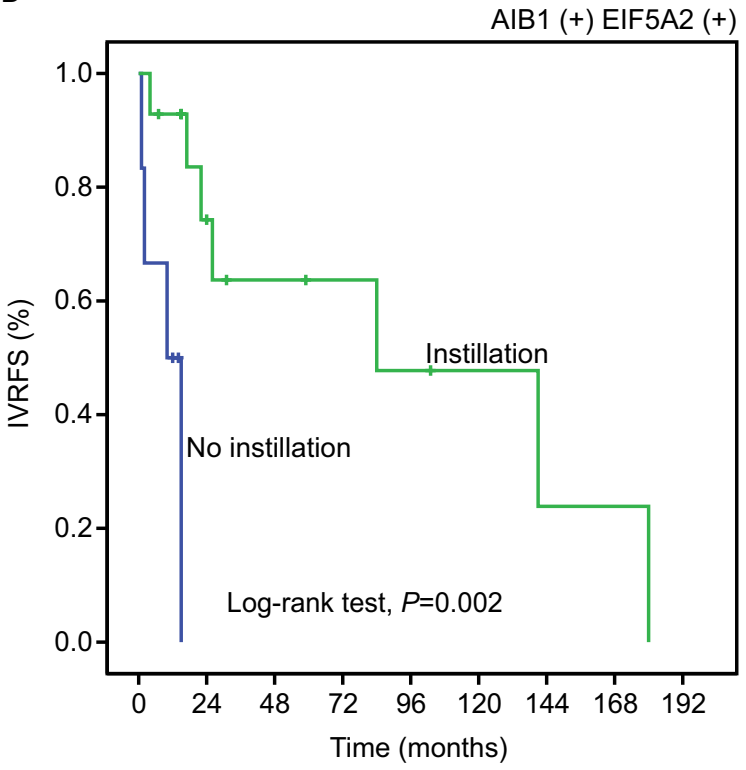

Figure 5 Kaplan-Meier survival analysis of postoperative intravesical chemotherapy in subsets of different expression of AIBI and EIF5A2 (log-rank test).

Notes: AIBI (-): low AIBI expression, AIBI(+): high AIBI expression, EIF5A2 (-): low EIF5A2 expression, and EIF5A2 (+): high EIF5A2 expression. (A) AIBI(-) EIF5A2(-), probability of IVRFS of intravesical chemotherapy for patients with UTUC: no instillation (blue line), $n=1$; instillation (green line), $n=29$. (B) AIBI (-) EIF5A2 (+), probability of IVRFS of intravesical chemotherapy for patients with UTUC: no instillation (blue line), $\mathrm{n}=5$; instillation (green line), $\mathrm{n}=23$. (C) AIBI (+)EIF5A2(-), probability of IVRFS of intravesical chemotherapy for patients with UTUC: no instillation (blue line), $n=10$; instillation (green line), $n=21$. (D) AIBI (+)EIF5A2(+), probability of IVRFS of intravesical chemotherapy for patients with UTUC: no instillation (blue line), $n=6$; instillation (green line), $n=14$.

Abbreviations: IVRFS, intravesical recurrence-free survival; UTUC, upper tract urothelial carcinoma.

of AIB1 and EIF5A2n were positively correlated with an ascending clinical stage of the tumor. ${ }^{13,17,33,34}$ In the stratified survival analyses of UTUC, the expressions of AIB1 and EIF5A2 were considered to correlate with IVRFS of different subsets of UTUC patients and found high expression simultaneously in patients with pT2-4 (16/20, $80 \%)$ or high grade $(13 / 20,65 \%)$. Thus, it is indicated that
AIB1 and EIF5A2 expressions have the potential to predict postoperative bladder recurrence of certain UTUC patients. Furthermore, intravesical chemotherapy was a positive predictor of IVRFS as evidence by Kaplan-Meier curves in the subset of both high-level AIB1 and EIF5A2 expression. The examination of AIB1 and EIF5A2 expression by IHC, therefore, could be used as a meaningful tool in 
Table 3 Univariate and multivariate Cox regression models predicting bladder recurrence

\begin{tabular}{|c|c|c|c|c|c|c|}
\hline \multirow[t]{2}{*}{ Variables } & \multicolumn{3}{|c|}{ Univariate } & \multicolumn{3}{|c|}{ Multivariate } \\
\hline & HR & $95 \% \mathrm{Cl}$ & $P$-value & HR & $95 \% \mathrm{Cl}$ & $P$-value \\
\hline Age $(\leq 66 />66$ years $)$ & 1.136 & $0.438-2.948$ & 0.793 & & & \\
\hline Sex (male/female) & 0.951 & $0.36 \mathrm{I}-2.505$ & 0.919 & & & \\
\hline Tumor site (left/right) & 0.440 & $0.153-1.267$ & 0.128 & & & \\
\hline Tumor location & & & 0.994 & & & \\
\hline Calix or pelvis & & & 1.000 & & & \\
\hline Ureter & 0.944 & $0.330-2.699$ & 0.915 & & & \\
\hline$>$ I site & 0.958 & $0.188-4.872$ & 0.959 & & & \\
\hline Hydronephrosis (no/yes) & 3.187 & $0.724-14.025$ & 0.125 & & & \\
\hline Type of surgery (open/laparoscopy) & 0.473 & $0.164-1.363$ & 0.166 & & & \\
\hline RNU (no/yes) & 0.369 & $0.126-1.084$ & 0.070 & 0.316 & $0.097-1.031$ & 0.056 \\
\hline $\mathrm{pT}$ stage (pTa-TI/pT2-T4) & 3.001 & $0.860-10.475$ & 0.085 & & & \\
\hline Pathological $\mathrm{N}$ stage ( $\mathrm{pNx}$ or $\mathrm{pN} 0 / \mathrm{pNI}, 2)$ & 6.021 & $2.118-17.116$ & $0.001 *$ & 4.654 & I.459-14.850 & $0.009 *$ \\
\hline Tumor grade (low/high) & 1.356 & $0.470-3.911$ & 0.573 & & & \\
\hline Intravesical chemotherapy (no/yes) & 0.353 & $0.120-1.037$ & 0.058 & & & \\
\hline Adjuvant chemotherapy (no/yes) & 2.644 & $0.948-7.372$ & 0.063 & & & \\
\hline AIBI expression (low/high) & 3.256 & $1.127-9.406$ & $0.029 *$ & 3.252 & $1.090-9.705$ & $0.034 *$ \\
\hline EIF5A2 expression (low/high) & 5.646 & $1.601-19.914$ & $0.007^{*}$ & 4.613 & $1.249-17.038$ & $0.022 *$ \\
\hline
\end{tabular}

Notes: *Statistically significant. No RNU, segmental ureterectomy and kidney-sparing surgery.

Abbreviation: RNU, radical nephroureterectomy.
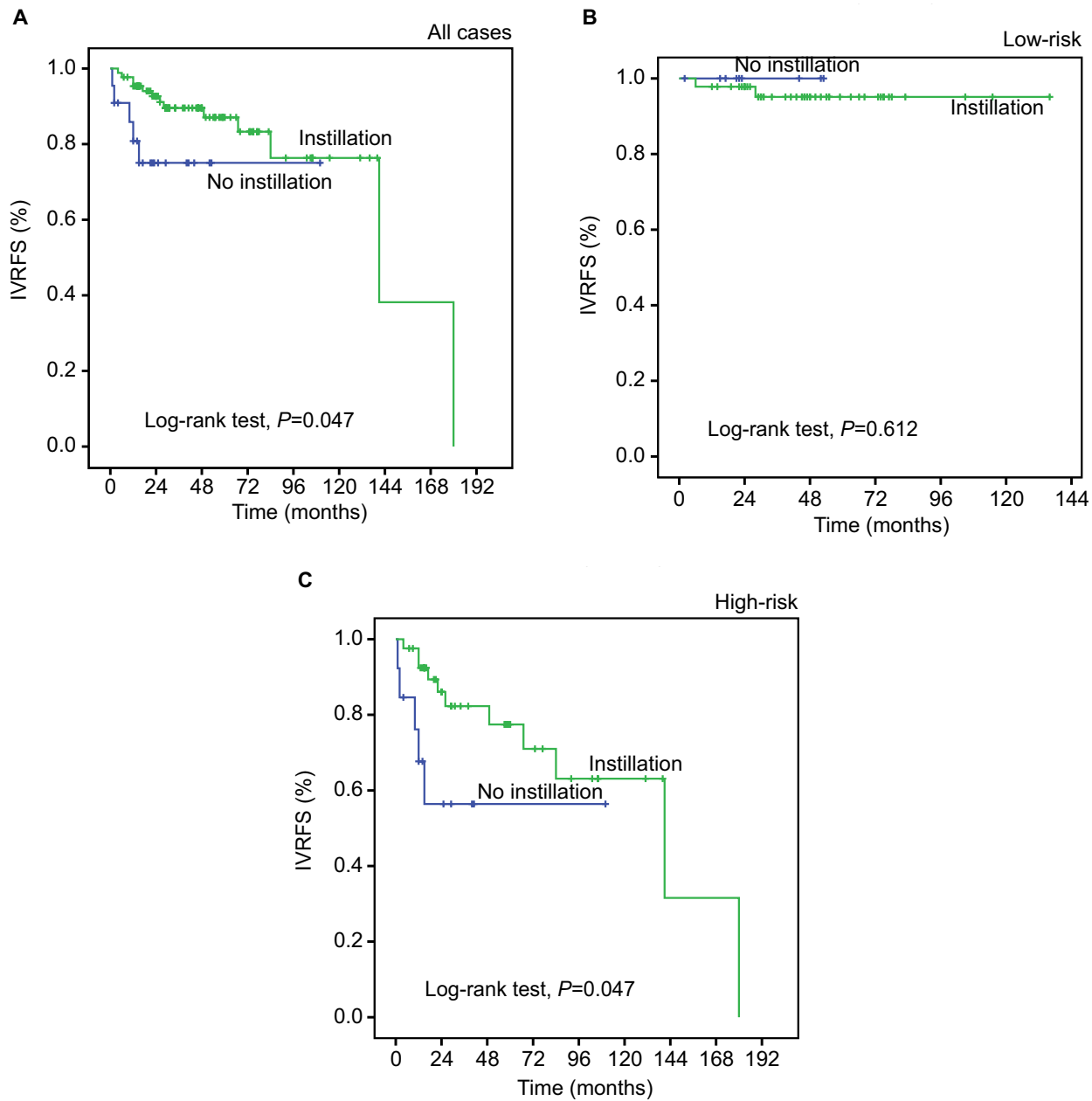

Figure 6 Kaplan-Meier survival analysis of postoperative intravesical chemotherapy in subsets of different risk groups of the prognostic model including ElF5A2, AIBI, lymph node status and RNU (log-rank test).

Note: Probability of IVRFS of all cases: no instillation (blue line), $n=22$; instillation (green line), $n=87$ (A); low-risk, probability of IVRFS of the low-risk group: no instillation (blue line), $n=9$; instillation (green line), $n=46$ (B); high-risk, probability of IVRFS of high-risk group: no instillation (blue line), $n=13$; instillation (green line), $n=4$ I (C).

Abbreviations: IVRFS, intravesical recurrence-free survival; RNU, radical nephroureterectomy. 


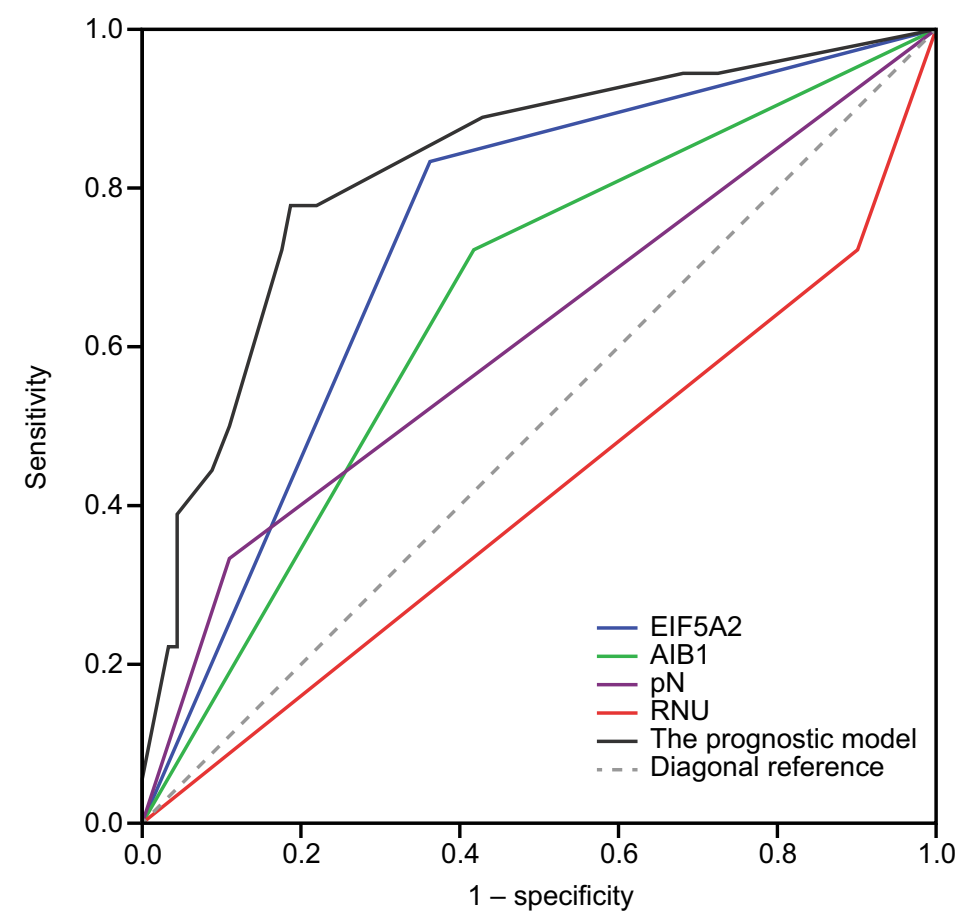

Figure 7 ROC curves comparing the predictive accuracy by the combined AIBI, EIF5A2, pN, and the surgical approach of RNU, the AIBI alone model, the EIF5A2 alone model, the $\mathrm{PN}$ model, and $\mathrm{RNU}$ alone model for intravesical recurrence-free survival.

Abbreviations: RNU, radical nephroureterectomy; ROC, receiver operating characteristic.

identifying those patients at the risk of bladder recurrence; AIB1 and EIF5A2 expression analysis may also be useful in optimizing postoperative individual UTUC therapy management. In other words, AIB1 and EIF5A may be considered as underlying biological biomarkers to predict the development of UTUC, and the combination of both markers shows more powerful predictive value of bladder recurrence in UTUC.

We noticed an informative correlation between intravesical recurrence of UTUC and surgery approach (RNU or not). Seisen et $\mathrm{a}^{35}$ demonstrated that survival was similar after KSS vs RNU only for low-grade and non-invasive UTUC, but in high-grade and invasive UTUC, RNU should be a standard treatment except some imperative cases, such as renal insufficiency or solitary functional kidney. In another study, Fang et $\mathrm{al}^{36}$ showed that no significant difference was found in terms of IVRFS (HR 1.35, $P=0.39$ ) between SU and (RNU in UTUC with favorable pathological features. However, these studies were subject to a selection bias favoring KSS inevitably. RNU with bladder cuff excision is still considered the standard treatment for UTUC and provides durable local control and favorable outcome in patients with localized UTUC. ${ }^{37}$ This opinion joins our study. Although the $P$-value only demonstrated a strong trend in univariate and multivariate analyses, we insisted that RNU should be a protective factor for intravesical recurrence.
Intravesical chemotherapy has been proven to reduce the risk of intravesical recurrence after surgery for UTUC in some large studies. We also demonstrated a promising association between this treatment and intravesical recurrence. However, about one-half of the patients with UTUC do not have intravesical recurrence in the subsequent years, ${ }^{3-5}$ so it indicates that the therapy strategy may be an excessive treatment for low-risk patients, and it is necessary to take individualized treatment according to a patient's clinical and pathological characteristics. Although an increasing number of studies have focused on risk factors for intravesical recurrence after RNU and investigated the association between the expression of some biomarkers, such as endoglin or Ki-67, the search for specific molecular markers for UTUC, which have clinical/prognostic significance for intravesical recurrence, remains substantially limited. ${ }^{38,39}$ In this study, we found a significant association between intravesical chemotherapy and the combination of AIB1 and EIF5A2. To date, the field cancerization hypothesis ${ }^{40,41}$ and intraluminal seeding ${ }^{42-44}$ have been the 2 main concepts to explain the mechanism of intravesical recurrence after surgery. In diverse human malignancies, AIB1 and EIF5A2 have been proven as putative oncogenes in tumorigenic processes, and so we surmise that they might involve the progression of UTUC. The subset group of UTUC patients with high level of AIB1 and EIF5A2 might be inclined to 
A

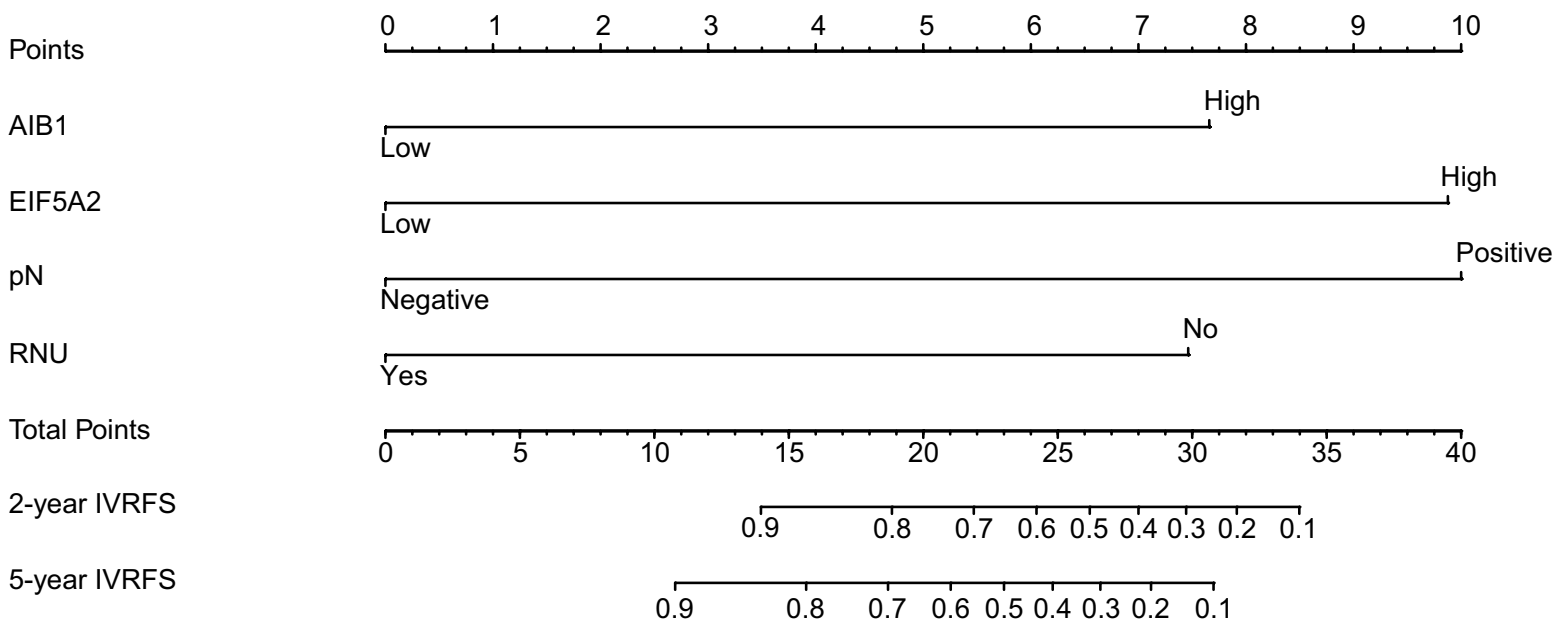

B

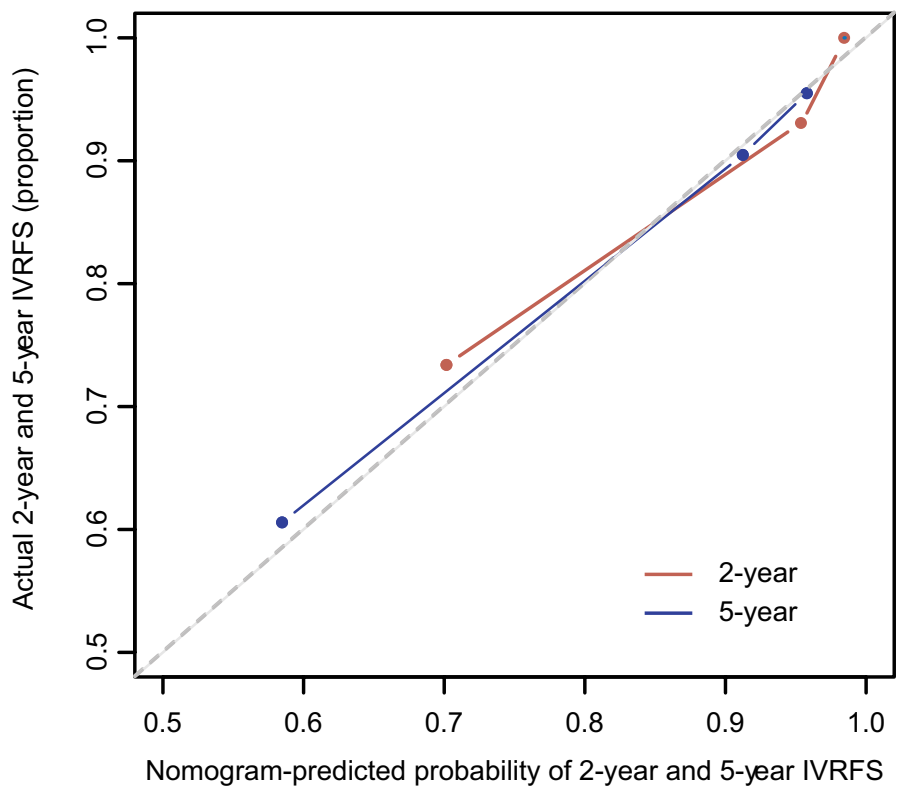

Figure $8 \mathrm{~A}$ nomogram to predict 2-year and 5-year IVRFS after surgery in patients with upper tract urothelial carcinoma (A). Calibration plots demonstrate virtually ideal predictions for 2-year and 5-year IVRFS (B).

Abbreviations: IVRFS, intravesical recurrence-free survival; RNU, radical nephroureterectomy.

implant dispersed intraluminal viable cancer cells or establish a significant new tumor, and intravesical chemotherapy should be more meaningful for this subset group of UTUC patients after surgery.

Recently, several studies have identified some potential outcome predictors for UTUC following surgery. ${ }^{45,46}$ Xylinas et $\mathrm{al}^{47}$ developed nomograms for predicting intravesical recurrence with some clinicopathological factors, which is useful to make informed medical decisions regarding management of their disease. Hashimoto et al considered gross hematuria and preoperative serum creatinine levels as independent predictors for intravesical recurrence after RNU and developed a nomogram based on these factors to guide patient selection for postoperative therapeutic interventions. ${ }^{48}$ Incorporating biologically significant biomarkers into the prediction model may allow us to select patients for 
administration of intravesical chemotherapy more accurately. In this study, the presence of AIB1, EIF5A2 and pN+ was found to be associated with an elevated risk for intravesical recurrence, and surgical approach with RNU was considered as a protective factor for intravesical recurrence. Further use of this prediction model developed with these 4 factors might allow for better identification of patients who classified as high risk of intravesical recurrence and who are most likely to benefit from intravesical chemotherapy. Meanwhile, we developed a nomogram for the prediction of intravesical recurrence after surgery. We found that our nomogram was well calibrated, as the slopes of the calibration curves were close to 1 . The use of our accurate, well-calibrated predictive model should improve clinicians' abilities to classify patients for postoperative therapeutic strategies, including prophylactic intravesical instillation.

This study has several limitations. First and foremost are the limitations inherent in the retrospective singlecenter study design. External validation of the current prognostic model is required before widespread application. In addition, the procedure of intravesical chemotherapy was done by several different surgeons, which accounts for some variability, especially in the selection of chemotherapeutics, thereby abrogating the effects seen in this study. ${ }^{49,50}$ Finally, the proportion of patients with postoperative intravesical instillation in the AIB1 high expression group was small, because of the uncontrollable retrospective data.

\section{Conclusion}

We describe, for the first time, that AIB1 and EIF5A2 expressions are associated with shorter IVRFS time in UTUC. We developed a prognostic model based on some clinicopathological factors, including AIB1 and EIF5A2, which predict intravesical recurrence after surgery with reasonable accuracy. The nomogram that was developed from the prognostic model may improve the clinical decision-making process regarding postoperative intravesical chemotherapy.

\section{Ethics approval and informed consent}

This study was approved by the medical ethics committee of our institute.

\section{Acknowledgment}

This work was supported by National Key Research and Development Program of China (Grant/Award Number:
2016YFC0902600), Guangdong Provincial Science and Technology Foundation (Grant/Award Number: 2014B020212015, 2017B020227004 and 2017A030313538), National Natural Science Foundation of China (Grant/Award Number: 81372357, 81725016 and 81572905), Guangdong Medical Science and Technology Research Foundation (Grant/Award Number: A2018040) and the Guangzhou Science and Technology Foundation (201607010238 and 201704020174).

\section{Disclosure}

The authors report no conflicts of interest in this work.

\section{References}

1. Rouprêt M, Zigeuner R, Palou J, et al. European guidelines for the diagnosis and management of upper urinary tract urothelial cell carcinomas: 2011 update. Eur Urol. 2011;59(4):584-594.

2. Gupta R, Paner GP, Amin MB. Neoplasms of the upper urinary tract: a review with focus on urothelial carcinoma of the pelvicalyceal system and aspects related to its diagnosis and reporting. Adv Anat Pathol. 2008;15(3):127-139.

3. Ito A, Shintaku I, Satoh M, et al. Prospective randomized phase II trial of a single early intravesical instillation of pirarubicin (THP) in the prevention of bladder recurrence after nephroureterectomy for upper urinary tract urothelial carcinoma: the THP Monotherapy Study Group Trial. J Clin Oncol. 2013;31(11):1422-1427.

4. Huang WW, Huang HY, Liao AC, et al. Primary urothelial carcinoma of the upper tract: important clinicopathological factors predicting bladder recurrence after surgical resection. Pathol Int. 2009;59(9):642-649.

5. Novara G, De Marco V, Dalpiaz O, et al. Independent predictors of metachronous bladder transitional cell carcinoma (TCC) after nephroureterectomy for TCC of the upper urinary tract. BJU Int. 2008;101(11):1368-1374.

6. Schrier BP, Hollander MP, van Rhijn BW, Kiemeney LA, Witjes JA. Prognosis of muscle-invasive bladder cancer: difference between primary and progressive tumours and implications for therapy. Eur Urol. 2004;45(3):292-296.

7. Scarpini S, Rouprêt M, Renard-Penna R, Camparo P, Cussenot O, Compérat E. Impact of the expression of Aurora-A, p53, and MIB-1 on the prognosis of urothelial carcinomas of the upper urinary tract. Urol Oncol.2012;30(2):182-187.

8. Krabbe LM, Bagrodia A, Lotan Y, et al. Prospective analysis of Ki-67 as an independent predictor of oncologic outcomes in patients with high grade upper tract urothelial carcinoma. J Urol. 2014;191(1):28-34.

9. Zhang X, Kong C, Takenaka I. Evaluation of cell proliferation, apoptosis, and angiogenesis in transitional cell carcinoma of the renal pelvis and ureter. Urology. 2001;57(5):981-985.

10. Anzick SL, Kononen J, Walker RL, et al. AIB1, a steroid receptor coactivator amplified in breast and ovarian cancer. Science. 1997;277(5328):965-968.

11. Liao L, Kuang SQ, Yuan Y, Gonzalez SM, O’Malley BW, Xu J. Molecular structure and biological function of the cancer-amplified nuclear receptor coactivator SRC-3/AIB1. J Steroid Biochem Mol Biol. 2002;83(1-5):3-14.

12. Gnanapragasam VJ, Leung HY, Pulimood AS, Neal DE, Robson CN. Expression of RAC 3, a steroid hormone receptor co-activator in prostate cancer. Br J Cancer. 2001;85(12):1928-1936.

13. Tanner MM, Grenman S, Koul A, et al. Frequent amplification of chromosomal region 20q12-q13 in ovarian cancer. Clin Cancer Res. 2000;6(5):1833-1839.

14. Ghadimi BM, Schröck E, Walker RL, et al. Specific chromosomal aberrations and amplification of the AIB1 nuclear receptor coactivator gene in pancreatic carcinomas. Am J Pathol. 1999;154(2):525-536.

15. Xu FP, Xie D, Wen JM, et al. SRC-3/AIB1 protein and gene amplification levels in human esophageal squamous cell carcinomas. Cancer Lett. 2007;245(1-2):69-74. 
16. Sakakura C, Hagiwara A, Yasuoka R, et al. Amplification and overexpression of the AIB1 nuclear receptor co-activator gene in primary gastric cancers. Int J Cancer. 2000;89(3):217-223.

17. Xie D, Sham JS, Zeng WF, et al. Correlation of AIB1 overexpression with advanced clinical stage of human colorectal carcinoma. Hum Pathol. 2005;36(7):777-783.

18. Luo JH, Xie D, Liu MZ, et al. Protein expression and amplification of AIB1 in human urothelial carcinoma of the bladder and overexpression of AIB1 is a new independent prognostic marker of patient survival. Int J Cancer. 2008;122(11):2554-2561.

19. Guan XY, Sham JS, Tang TC, Fang Y, Huo KK, Yang JM. Isolation of a novel candidate oncogene within a frequently amplified region at $3 \mathrm{q} 26$ in ovarian cancer. Cancer Res. 2001;61(9):3806-3809.

20. Marchet A, Mocellin S, Belluco C, et al. Gene expression profile of primary gastric cancer: towards the prediction of lymph node status. Ann Surg Oncol. 2007;14(3):1058-1064.

21. Zhu W, Cai MY, Tong ZT, et al. Overexpression of EIF5A2 promotes colorectal carcinoma cell aggressiveness by upregulating MTA1 through C-myc to induce epithelial-mesenchymaltransition. Gut. 2012;61(4):562-575.

22. Tang DJ, Dong SS, Ma NF, et al. Overexpression of eukaryotic initiation factor 5A2 enhances cell motility and promotes tumor metastasis in hepatocellular carcinoma. Hepatology. 2010;51(4):1255-1263.

23. Yang GF, Xie D, Liu JH, et al. Expression and amplification of eIF-5A2 in human epithelial ovarian tumors and overexpression of EIF-5A2 is a new independent predictor of outcome in patients with ovarian carcinoma. Gynecol Oncol. 2009;112(2):314-318.

24. He LR, Zhao HY, Li BK, et al. Overexpression of eIF5A-2 is an adverse prognostic marker of survival in stage I non-small cell lung cancer patients. Int J Cancer. 2011;129(1):143-150.

25. Chen W, Luo JH, Hua WF, et al. Overexpression of EIF-5A2 is an independent predictor of outcome in patients of urothelial carcinoma of the bladder treated with radical cystectomy. Cancer Epidemiol Biomarkers Prev. 2009;18(2):400-408.

26. Catto JW, Azzouzi AR, Amira N, et al. Distinct patterns of microsatellite instability are seen in tumours of the urinary tract. Oncogene. 2003;22(54):8699-8706.

27. Roupret M, Catto J, Coulet F, et al. Microsatellite instability as indicator of MSH2 gene mutation in patients with upper urinary tract transitional cell carcinoma. J Med Genet. 2004;41(7):e91.

28. Jou YC, Tsai YS, Chen SY, Hsieh HY, Tsai HT, Tzai TS. Loss of DAB2IP expression in human urothelial carcinoma is associated with poorer recurrence-free survival. Virchows Arch. 2016;468(6):733-740.

29. Hall MC, Womack S, Sagalowsky AI, Carmody T, Erickstad MD, Roehrborn CG. Prognostic factors, recurrence, and survival in transitional cell carcinoma of the upper urinary tract: a 30-year experience in 252 patients. Urology. 1998;52(4):594-601.

30. Green DA, Rink M, Xylinas E, et al. Urothelial carcinoma of the bladder and the upper tract: disparate twins. J Urol. 2013;189(4):1214-1221.

31. Siegel RL, Miller KD, Jemal A. Cancer statistics, 2016. CA Cancer J Clin. 2016;66(1):7-30.

32. Tong ZT, Wei JH, Zhang JX, et al. AIB1 predicts bladder cancer outcome and promotes bladder cancer cell proliferation through AKT and E2F1. Br J Cancer. 2013;108(7):1470-1479.

33. Guan XY, Fung JM, Ma NF, et al. Oncogenic role of eIF-5A2 in the development of ovarian cancer. Cancer Res. 2004;64(12):4197-4200.

34. Xie D, Ma NF, Pan ZZ, et al. Overexpression of EIF-5A2 is associated with metastasis of human colorectal carcinoma. Hum Pathol. 2008;39(1): 80-86.
35. Seisen T, Peyronnet B, Dominguez-Escrig JL, et al. Oncologic Outcomes of Kidney-sparing Surgery Versus Radical Nephroureterectomy for Upper Tract Urothelial Carcinoma: A Systematic Review by the EAU Non-muscle Invasive Bladder Cancer Guidelines Panel. Eur Urol. 2016;70(6):1052-1068.

36. Fang D, Seisen T, Yang K, et al. A systematic review and meta-analysis of oncological and renal function outcomes obtained after segmental ureterectomy versus radical nephroureterectomy for upper tract urothelial carcinoma. Eur J Surg Oncol. 2016;42(11):1625-1635.

37. Margulis V, Shariat SF, Matin SF, et al; Upper Tract Urothelial Carcinoma Collaboration. Outcomes of radical nephroureterectomy: a series from the Upper Tract Urothelial Carcinoma Collaboration. Cancer. 2009;115(6):1224-1233.

38. Wu P, Liu S, Zhang W, et al. Low-level Ki-67 expression as an independent predictor of bladder tumour recurrence in patients with primary upper tract urothelial carcinoma after radical nephroureterectomy. Jpn J Clin Oncol. 2015;45(12):1175-1181.

39. Fujita K, Ujike T, Nagahara A, et al. Endoglin expression in upper urinary tract urothelial carcinoma is associated with intravesical recurrence after radical nephroureterectomy. Int J Urol. 2015;22(5):463-467.

40. Miyake H, Hara I, Kamidono S, Eto H. Multifocal transitional cell carcinoma of the bladder and upper urinary tract: molecular screening of clonal origin by characterizing CD44 alternative splicing patterns. J Urol. 2004;172(3):1127-1129.

41. Millán-Rodríguez F, Chéchile-Toniolo G, Salvador-Bayarri J, HuguetPérez J, Vicente-Rodríguez J. Upper urinary tract tumors after primary superficial bladder tumors: prognostic factors and risk groups. J Urol. 2000;164(4):1183-1187.

42. Habuchi T, Takahashi R, Yamada H, Kakehi Y, Sugiyama T, Yoshida O. Metachronous multifocal development of urothelial cancers by intraluminal seeding. Lancet. 1993;342(8879):1087-1088.

43. Hafner C, Knuechel R, Zanardo L, et al. Evidence for oligoclonality and tumor spread by intraluminal seeding in multifocal urothelial carcinomas of the upper and lower urinary tract. Oncogene. 2001;20(35):4910-4915.

44. Catto JW, Hartmann A, Stoehr R, et al. Multifocal urothelial cancers with the mutator phenotype are of monoclonal origin and require panurothelial treatment for tumor clearance. J Urol. 2006;175(6):2323-2330.

45. Chromecki TF, Bensalah K, Remzi M, et al. Prognostic factors for upper urinary tract urothelial carcinoma. Nat Rev Urol. 2011;8(8):440-447.

46. Lughezzani G, Burger M, Margulis V, et al. Prognostic factors in upper urinary tract urothelial carcinomas: a comprehensive review of the current literature. Eur Urol. 2012;62(1):100-114.

47. Xylinas E, Kluth L, Passoni N, et al; UTUC Collaboration. Prediction of intravesical recurrence after radical nephroureterectomy: development of a clinical decision-making tool. Eur Urol. 2014;65(3):650-658.

48. Hashimoto T, Nakashima J, Kashima T, et al. Clinical significance of preoperative renal function and gross hematuria for intravesical recurrence after radical nephroureterecto my for upper tract urothelial carcinoma. Int J Urol. 2017;24(2):111-116.

49. Xylinas E, Rink M, Cha EK, et al; Upper Tract Urothelial Carcinoma Collaboration. Impact of distal ureter management on oncologic outcomes following radical nephroureterectomy for upper tract urothelial carcinoma. Eur Urol. 2014;65(1):210-217.

50. O’Brien T, Ray E, Singh R, Coker B, Beard R; British Association of Urological Surgeons Section of Oncology. Prevention of bladder tumours after nephroureterectomy for primary upper urinary tract urothelial carcinoma: a prospective, multicentre, randomised clinical trial of a single postoperative intravesical dose of mitomycin $\mathrm{C}$ (the ODMIT-C Trial). Eur Urol. 2011;60(4):703-710. 


\section{Supplementary material}

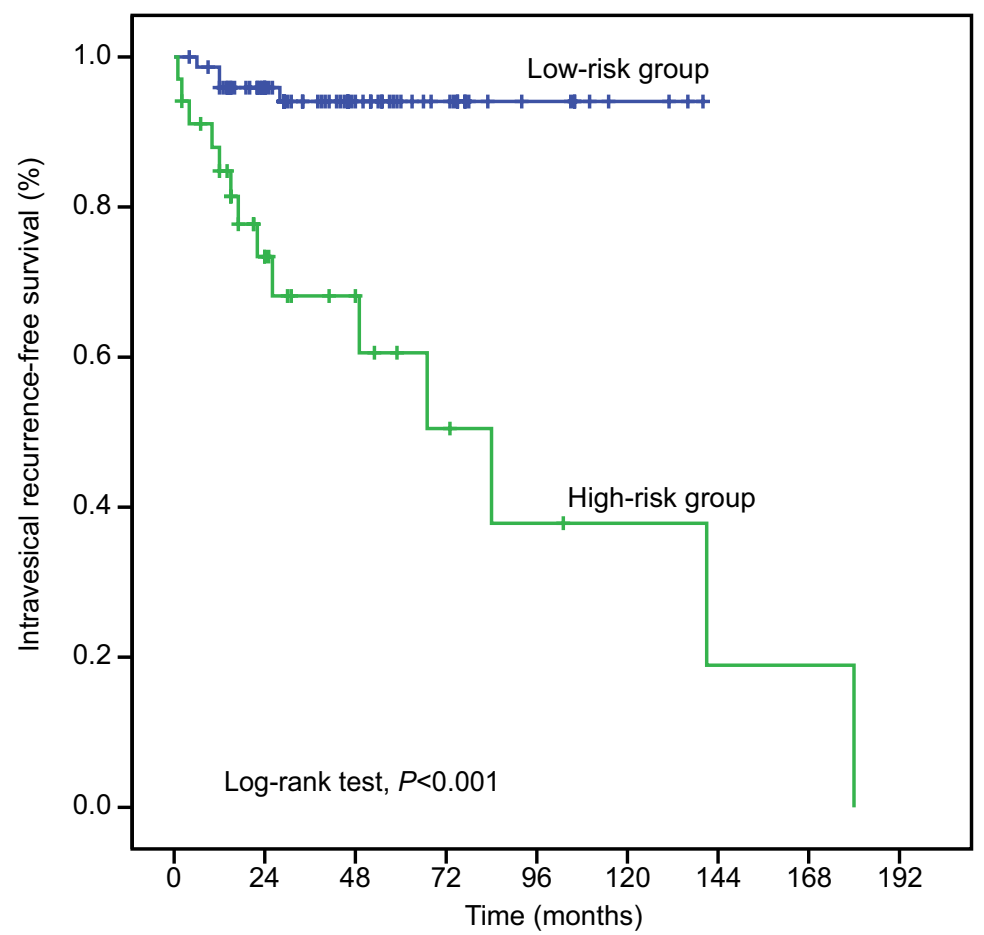

Figure SI Kaplan-Meier plot shows intravesical recurrence-free survival curves stratified by 4 independent prognostic factors (EIF5A2, AIBI, lymph node status and RNU). Note: Low-risk group (blue line), $n=75$; high-risk group (green line), $n=34$.

Abbreviation: RNU, radical nephroureterectomy.

Cancer Management and Research is an international, peer-reviewed open access journal focusing on cancer research and the optimal use of preventative and integrated treatment interventions to achieve improved outcomes, enhanced survival and quality of life for the cancer patient. The manuscript management system is completely online and includes

\section{Dovepress}

a very quick and fair peer-review system, which is all easy to use. Visit http://www.dovepress.com/testimonials.php to read real quotes from published authors.

Submit your manuscript here: https://www.dovepress.com/cancer-management-and-research-journal 